\title{
Resource allocation for psychosis in Australia
}

\author{
Vaughan J. Carr ${ }^{1}$, Amanda L Neil ${ }^{2}$ and Terry J. Lewin ${ }^{3}$
}

1Professor of Psychiatry, Centre for Mental Health Studies, University of Newcastle, Callaghan, NSW 2308, Australia, email Vaughan.Carr@hunter.health.nsw.gov.au; Director, Hunter Mental Health; Scientific Director, Neuroscience Institute of Schizophrenia and Allied Disorders (NISAD)

2Senior Lecturer in Health Economics, Centre for Clinical Epidemiology and Biostatistics, University of Newcastle ${ }^{3}$ Research Manager, Hunter Mental Health and the Centre for Mental Health Studies

sing a census-based prevalence survey (Jablensky et al, 2000), we estimated the cost of psychosis in urban Australia at AU $\$ 2.25$ billion ( $£ 0.86$ billion) per year when valued at prices pertaining in the year 2000 (Carr et al, 2003). About $40 \%$ of these costs were spent on direct mental health care, the remainder being the costs of lost productivity (limited to unemployment in our study). The total costs amounted to AU \$46 200 (£17 722) per person per year, $20 \%$ higher than the average annual male income. The bulk of the treatment cost was accounted for by in-patient care, which appeared to have become the default option in the absence of adequate levels of supported community accommodation. This was indicated by the fact that after 'non-discretionary' treatment costs ( $42 \%$ of direct costs) were accounted for (i.e. visits to a general practitioner, medication, crisis or emergency care, acute hospitalisation), almost three-quarters of the remainder was spent on long-stay hospitalisation ( $\mathrm{N}$ eil et al, 2003). When patterns of communitybased service delivery were examined, we found a marked paucity of delivery of psychosocial treatments, rehabilitation and substance use interventions, reflecting the skewing of expenditure towards long-term hospitalisation and away from community care.

This does not look like good value for money. Indeed, attention has been drawn to the fact that the burden due to schizophrenia that is averted by current treatment practices is low, in spite of the high cost. Modest but significant gains in burden averted could be achieved with better deployment of evidence-based treatments, and with almost double the cost-effectiveness (Andrews et al, 2003, 2004). Thus, there is an opportunity cost in maintaining the status quo.

The question, then, is how can the existing 'discretionary' resources (i.e. non-primary care, nonemergency care and long-stay hospitalisation) be reallocated from their current pattern of distribution for the psychoses to ensure wider delivery of evidence-based community treatments and improved outcomes, with greater cost-effectiveness?

We argue that the starting point ought not to be with relativities in disease burden but with the identification of evidence-based interventions that are efficient (i.e. effective, good value for money and affordable), subject to equity and feasibility considerations ( $\mathrm{N}$ eil et al, 2003).

\section{Efficiency}

In the absence of pragmatic economic trials, the determination of treatment efficiency is likely to proceed through several steps, from the assessment of treatment efficacy, to treatment and programme effectiveness, to cost-effectiveness and likely societal benefits. The identification of effective treatments first entails a review of efficacy studies of 'experimental' treatments based on randomised controlled trials, the 'gold standard' of efficacy measurement. Examples in schizophrenia are: antipsychotic drugs, family-based interventions (which include psychoeducation and the development of problemsolving skills); cognitive-behavioural treatments for better psychotic symptom control, relapse prevention and medication adherence; assertive community treatment; and supported employment programmes. Second, clinical effectiveness studies are needed - that is, systematic, non-experimental investigations of the effectiveness of such treatments as the foregoing in ordinary clinical settings, with the determination of clinical outcomes and costs. Where they are lacking, services ought to conduct these evaluations themselves. This has the advantage of staff being trained to deliver evidence-based treatments, to conduct appropriate clinical measurements, and to assess outcomes. In turn, this has a cascade effect, with other staff seeking to learn the treatment and measurement techniques. Subsequently, these skills are likely to generalise to other areas of their clinical work as a culture of evidence-based practice and clinical measurement begins to take hold. Third, findings from service systems research need to be examined, for example comparisons between community $v$. residential treatment, primary health care $v$. treatment in specialist settings, and separate v. integrated substance misuse services for those with dual diagnoses, with outcomes measured in terms of readmission rates, referral patterns and so on.

Estimations of the likely cost-effectiveness of such interventions constitute the next step in the process; the additional benefits must be worth the additional costs if these interventions are to represent value for money. Economic evaluations entail analyses of the costs and consequences of an intervention compared with those of an appropriate comparator. The evaluation techniques used (e.g. cost-benefit, cost-utility, cost-effectiveness, and cost-minimisation analyses) primarily depend on the available range of outcome measures and the differences in outcomes across the various treatment arms. Ideally, economic evaluations should be context specific and
Attention has been drawn to the fact that the burden due to schizophrenia that

is averted by

current treatment practices is low, in spite of the high cost. Modest but significant gains in

burden averted could be achieved with better deployment of evidence-based treatments, and with almost double the costeffectiveness. 
employ a societal perspective in addition to any other perspective utilised (e.g. individual, third-party payer, government).

Affordability is simply the question of whether the available resources are sufficient to meet the costs of implementing and maintaining the proposed interventions.

Cost modelling studies do not directly address the above issues, but they can help identify the main drivers of costs and assess the effect of interventions on broader costs (e.g. Carr et al, 2004).

\section{There is a demonstrably high level of expenditure on psychosis in Australia and a more rational basis for resource allocation is required, driven primarily by treatment efficiency and equity considerations.}

\section{Equity and feasibility}

The principle of equity has to do with the extent to which a given society may seek preferential allocation of resources for socially and economically disadvantaged groups, marginalised or less powerful groups, remote or isolated communities, and diseases that may be conspicuous in the community or associated with high levels of disability (e.g. psychoses). Feasibility refers to whether the intervention falls within the existing or readily achievable range of human expertise or technological capacity.

\section{Other issues}

H aving thus established a priority list of potential evidencebased, cost-effective interventions, assessed their affordability, and addressed questions of equity and feasibility, there are further issues to be addressed. Structural adjustments are necessary to permit the flexible allocation of 'discretionary' expenditure (e.g. in Australia, shifting from long-term hospitalisation to supported community accommodation in the treatment of psychosis). 0 ther implementation issues include training, administration, uptake by clinicians, and intangible or hidden costs. Programmes are necessary to increase clinicians' and administrators' awareness of efficient interventions, to improve the therapeutic skills of clinicians, and to motivate them to provide the identified interventions. Motivation to deliver the interventions can be enhanced through the provision of incentives. These may include a combination of financial rewards and disincentives, prestige enhancement or promotion, performance reviews, and feedback of information concerning effectiveness and efficiency. Implementation should also be monitored at the individual, health system and government levels so that timely adjustments can be made.

\section{Conclusions}

There is a demonstrably high level of expenditure on psychosis in Australia and a more rational basis for resource allocation is required, driven primarily by treatment efficiency and equity considerations. We also contend that many of the principles outlined above are not just applicable to the psychoses but could usefully inform decisions about resource distribution in mental health services generally.

\section{References}

Andrews, G., Sanderson K., Corry, J., et al (2003) Costeffectiveness of current and optimal treatment for schizophrenia. British Journal of Psychiatry, 183, 427-435.

Andrews, G., Issakidis, C., Sanderson K., et al (2004) Utilising survey data to inform public policy: comparison of the costeffectiveness of treatment of ten mental disorders. British Journal of Psychiatry, 184, 526-533.

Carr, V. J., N eil, A. L., Halpin, S. A., et al (2003) Costs of schizophrenia and other psychoses in urban Australia: findings from the Low Prevalence (psychotic) Disorders Study. Australian and New Zealand Journal of Psychiatry, 37, 31-40.

Carr, V. J., Lewin, T. J., N eil, A. L., et al (2004) Premorbid, psychosocial and clinical predictors of the costs of schizophrenia and other psychoses. British Journal of Psychiatry, 184, 517-525.

ablensky, A., McGrath, J., Herrman, H., et al (2000) Psychotic disorders in urban areas: an overview of the methods and findings of the study on low prevalence disorders. Australian and New Zealand Journal of Psychiatry, 34, 221236

$\mathrm{N}$ eil, A. L., Lewin, T. J. \& Carr, V. J. (2003) Allocation of resources and psychosis. Australian and $\mathrm{New} Z$ Zealand Journal of Psychiatry, 37, 15-23.

\section{Psychiatric senvices in the fifth year of health care reform in Poland}

\section{Wanda Langiewicz and Elzbieta Slupczynska-Kossobudzka}

Department of Health Care Organisation, Institute of Psychiatry and Neurology, Warsaw, Poland, email langiew@ipin.edu.pl

hanges in the Polish health care system, introduced by a Parliamentary Act in 1999, resulted from an urgent need for a more effective provision of health services, which were held in poor esteem by the public. Public expenditure on health care at the time of the reform was equivalent to $4.19 \%$ of gross national product, or US\$363 at purchasing power parity (PPP) per capita. This amount was considerably lower than in the most developed countries (i.e. members of the Organisation for 